\title{
A new locus for split hand/foot malformation with long bone deficiency (SHFLD) at 2q14.2 identified from a chromosome translocation
}

\author{
Christian Babbs · Raoul Heller • David B. Everman • \\ Mark Crocker · Stephen R. F. Twigg · Charles E. Schwartz • \\ Henk Giele • Andrew O. M. Wilkie
}

Received: 16 April 2007 / Accepted: 18 May 2007 / Published online: 14 June 2007

(C) Springer-Verlag 2007

\begin{abstract}
Split hand/foot malformation (SHFM) with long bone deficiency (SHFLD) is a distinct entity in the spectrum of ectrodactylous limb malformations characterised by associated tibial a/hypoplasia. Pedigrees with multiple individuals affected by SHFLD often include nonpenetrant intermediate relatives, making genetic mapping difficult. Here we report a sporadic patient with SHFLD who carries a de novo chromosomal translocation $\mathrm{t}(2 ; 18)(\mathrm{q} 14.2 ; \mathrm{p} 11.2)$. Characterisation of the breakpoints revealed that neither disrupts any known gene; however, the chromosome 2 breakpoint lies between GLI2 and $I N H B B$, two genes known to be involved in limb development. To investigate whether mutation of a gene in proxim-
\end{abstract}

The authors Christian Babbs and Raoul Heller have contributed equally to this study.

C. Babbs · R. Heller · S. R. F. Twigg · A. O. M. Wilkie (凹)

Weatherall Institute of Molecular Medicine,

University of Oxford, John Radcliffe Hospital, Oxford, UK

e-mail: awilkie@hammer.imm.ox.ac.uk

D. B. Everman - C. E. Schwartz

Center for Molecular Studies,

JC Self Research Institute for Human Genetics,

Greenwood, SC USA

M. Crocker

Medical Genetics Laboratories,

Churchill Hospital, Oxford, UK

H. Giele

Department of Plastic and Reconstructive Surgery,

John Radcliffe Hospital, Oxford, UK

Present Address:

R. Heller

Institute of Human Genetics,

University Hospital of Cologne, Cologne, Germany ity to the chromosome 2 breakpoint underlies the SHFLD, we sought independent evidence of mutations in GLI2, $I N H B B$ and two other genes (RALB and FLJ14816) in 44 unrelated patients with SHFM, SHFLD or isolated long bone deficiency. No convincing pathogenic mutations were found, raising the possibility that a long-range cis acting regulatory element may be disrupted by this translocation. The previous description of a translocation with a $2 q 14.2$ breakpoint associated with ectrodactyly, and the mapping of the ectrodactylous Dominant hemimelia mouse mutation to a region of homologous synteny, suggests that $2 \mathrm{q} 14.2$ represents a novel locus for SHFLD.

\section{Introduction}

Ectrodactyly or split hand/foot malformation (SHFM) has a birth prevalence of $\sim 1$ in 18,000 (Czeizel et al. 1993) and presents in a variable fashion. The archetypal form involves deficiency of the central rays of the hands and feet, characterised by median clefts associated with syndactyly of the remaining digits and aplasia and/or hypoplasia of the phalanges, metacarpals and metatarsals (MIM 183600). Alternatively oligo- or monodactyly may occur, often in association with hemimelic deficiency of the distal long bones of the upper or lower limb, with the radius and tibia being more frequently involved. This variation may be found in the same pedigree or even on different limbs of a single individual.

Isolated SHFM is genetically heterogeneous and five loci have been mapped: SHFM1 on chromosome 7q21 (MIM 183600), SHFM2 on Xq26 (MIM 313350), SHFM3 on chromosome 10q24 (MIM 600095), SHFM4 on chromosome 3q27 (MIM 605289), and SHFM5 on chromosome $2 q 31$ (606708). Of these, only intragenic mutations in the 
TP73L gene encoding the P63 protein (on 3q27) have been identified as a relatively rare cause of SHFM (Ianakiev et al. 2000; van Bokhoven et al. 2001). TP73L mutations more frequently cause EEC syndrome (MIM 129900), which includes ectrodactyly, ectodermal dysplasia and cleft lip/palate (Celli et al. 1999; de Mollerat et al. 2003a). More commonly, complex chromosome rearrangements seem to underlie SHFM. The 7q21 locus is associated with both deletions and duplications, candidate genes are $D L X 5$, DLX6 and DSS1 (Scherer et al. 1994; Crackower et al. 1996), the 10q24 locus is thought to involve rearrangements of the FBXW4 gene encoding DACTYLIN (Ianakiev et al. 1999; Sidow et al. 1999; de Mollerat et al. 2003b) and 2q31 is associated with deletions of the HOXD cluster, although the $D L X 1$ and $D L X 2$ genes are also candidates (Del Campo et al. 1999; Goodman et al. 2002).

Split hand/foot malformation associated with tibial a/hypoplasia appears to be a distinct syndrome that has been termed split hand/foot malformation with long bone deficiency (SHFLD) (MIM 19100), or alternatively SHFM type II (Zlotogora 1994) and is particularly disabling owing to the associated tibial deficiency which often requires belowknee amputation. Approximately 50 families and more than 100 sporadic cases of SHFLD have been described: the largest study reported 6 families and reviewed a further 31 with more than one affected member (Majewski et al. 1985) and found the phenotype to be very variable in severity, ranging from tetramonodactyly or transverse hemimelia to hypoplastic halluces. In most cases SHFLD follows an autosomal dominant pattern of inheritance. In some families, however, all affected individuals belong to the same generation suggesting autosomal recessive inheritance, although parental consanginuity has been noted in only one instance (Emami-Ahari and Mahloudji 1974). Furthermore, there is evidence for $\mathrm{X}$-linked recessive inheritance in one family (Kapur et al. 1982).

Significantly, when "pure" familial SHFM occurs, penetrance is at least $96 \%$ whereas in families in which at least one individual has additional long bone malformations (SHFLD), transmission is often unpredictable with penetrance among offspring of affected individuals being around $66 \%$; several pedigrees have been described in which related SHFLD cases are connected via multiple unaffected intermediate relatives (Majewski et al. 1985; Zlotogora 1994). Although both SHFM and SHFLD are likely to result from similar developmental mechanisms, the marked difference in inheritance patterns suggests that these malformations are distinct entities. This complicates the classification of sporadic cases of SHFM as it is unclear whether a given case is SHFM, or SHFLD with mild expressivity.

Recently, the first two specific loci for SHFLD have been proposed at chromosomes 1q42.2-q43 and 6q14.1, based on genome wide linkage analysis of a single large family (Naveed et al. 2007). Here we report a patient with SHFLD and who carries a de novo chromosomal translocation $\mathrm{t}(2 ; 18)(\mathrm{q} 14.2 ; \mathrm{p} 11.2)$. Initially, two pieces of evidence suggested that the $2 \mathrm{q} 14.2$ breakpoint might signal a locus for SHFLD. First, the report of a patient with a $\mathrm{t}(2 ; 4)(\mathrm{q} 14.2 ; \mathrm{q} 35)$ translocation associated with holoprosencephaly and ectrodactyly suggested a common aetiology (Corona-Rivera et al. 2000). Second, deletions of the short arm of chromosome 18 are relatively frequent and are not associated with limb abnormalities while confirmed deletions of 2 q14.2 are extremely rare (Schinzel 2001). We therefore characterised the breakpoints of the translocation and found that the $2 \mathrm{q} 14.2$ breakpoint lies between two genes, INHBB and GLI2 that have both been implicated in limb development. Moreover the breakpoint coincides with the homologous region of the mouse to which the Dominant hemimelia $(D h)$ limb malformation mutant has been mapped (Hughes et al. 1997). We therefore screened the four genes (GLI2, INHBB, RALB and FLJ14816) in proximity to the $2 q$ breakpoint for mutations in 44 patients with limb malformations including SHFM, SHFLD or isolated long bone deficiency; however, no clearly pathogenic mutation was found. The conserved synteny observed in a wide range of vertebrates across this region raises the possibility that a long-range cis acting regulatory region may be disrupted by this translocation.

\section{Methods}

Permission to undertake this work was obtained from the Oxfordshire Research Ethics Committee (reference: C99.181) and local institutional review boards. We ascertained the translocation case during a cohort study of patients requiring surgery for limb malformations. We screened an additional panel of 44 patients with SHFM or SHFLD. Of these ten were recruited from the Oxford study and included one case with SHFLD, seven with SHFM and two with long bone deficiency. All cases were sporadic except for one SHFM. The panel also included 25 patients ascertained during a previous study and in whom mutation analysis of $T P 73 L$ was negative (de Mollerat et al. 2003a), and 6 additional cases. These 31 patients included 17 cases of SHFLD ( 7 familial and 10 sporadic), 13 cases of SHFM ( 2 familial and 11 sporadic) and a single case of long bone deficiency. Some of the SHFLD cases do not have tibial deficiency but rather a/hypoplasia of another long bone, additionally four of the sporadic SHFM cases are syndromic including three with features overlapping EEC syndrome and one with Karsch-Neugebauer syndrome (MIM 183800). Three further sporadic cases of SHFLD ascertained during a separate study (Celli et al. 1999) were also screened. 
Fluorescence in situ hybridisation (FISH) mapping of the breakpoint used bacterial artificial chromosomes (BACs) obtained from Invitrogen. The chromosome 2 BACs were RP11-81B17, RP11-41C17, RP11-85B19, RP11-438012, RP11-56H11, RP11-78E20, RP11-1105G24 and RP11-416H1; the chromosome 18 BACs were RP11341P8, RP11-119J17, RP11-24G5 and RP11-976N2 (http:// www.genome.ucsc.edu/cgi-bin/hgGateway). The centromere of chromosome 18 was visualised using a specific biotinylated probe (Cambio) and detected with Texas Red. Probe generated from BAC clones was labelled with digoxygenin and detected with fluorescein.

To isolate the translocation breakpoint, single copy probes from the breakpoint spanning BAC RP11-56H11 were synthesised and hybridised to Southern blots of patient and control DNA. Initially, a $1.7 \mathrm{~kb} B s r$ GI breakpoint fragment was identified and this enabled further localisation on Southern blots by looking for additional breakpoint fragments. Having localised the der(18) breakpoint to a $55 \mathrm{bp}$ region, the breakpoint fragment was obtained by inverse PCR. Briefly, a BamHI fragment was identified as spanning the breakpoint. Genomic DNA from the patient was digested with BamHI, diluted to $10 \mathrm{ng} / \mu \mathrm{l}$ and T4 ligase was added to promote intramolecular ligation. Religated DNA was subjected to digestion with a second enzyme (Tsp45I) predicted to linearise the fragment and PCR was conducted using the primer pair: 5'-CC ATCCGTAGTCCCATGCTGAGG-3' and 5'-TAGGGAA TGGAAGGCGATGGCTCC-3'. DNA sequencing revealed 570 bp of unknown sequence, which was shown to originate from chromosome 18 by BLAST analysis. PCR with primers located centromeric to the $2 \mathrm{q}$ breakpoint and telomeric to the $18 \mathrm{p}$ breakpoint was employed to isolate the reciprocal breakpoint from the der(2). The primer pair used to amplify the der(2) breakpoint was 5'-AGTCTTCACA CAATCCAGCCACC-3' and 5'-AAGGATAGATTGGCA GGGCATC-3' and the primer pair for the der(18) breakpoint was 5'-GCACCTCGTTCTGTAAGCAGTCC-3' and 5'-CCATGCAGAGAAACATTAGCGTC-3'.

Array comparative genomic hybridisation (array CGH) was performed using a human $185 \mathrm{~K}$ genome-wide oligonucleotide array (Agilent Technologies) according to manufacturer's instructions. In brief, genomic DNA from the translocation patient and from a sex-matched reference were separately double-digested using the restriction endonucleases $A l u \mathrm{I}$ and $R s a \mathrm{I}$ (Promega) and purified using Microcon centrifugal filter devices (Millipore Corporation). A total of $1.5 \mu \mathrm{g}$ of the digested products was differentially labeled by the random priming method using the fluorophores Cy3-dUTP and Cy5-dUTP (Perkin Elmer) and cohybridised to the array for $48 \mathrm{~h}$ at $65^{\circ} \mathrm{C}$ in a rotating oven. The hybridised arrays were washed and scanned using an Agilent Microarray Scanner. The image data were extracted using Agilent Feature Extraction software version 8.5 and the data analysed using Agilent CGH Analytics software version 3.4 ( $z$-score method setting). These data have been submitted to the DECIPHER database (http://www.sanger. ac.uk/PostGenomics/decipher/).

The entire open reading frames of INHBB, GLI2, RALB and the predicted gene FLJ14816 were screened in the patient panel. Amplification was carried out in $30 \mu \mathrm{l}$ reactions containing $20 \mathrm{ng}$ of DNA, $1 \times$ Gold Buffer II (Applied Biosystems), $1.5 \mathrm{mM} \mathrm{MgCl}_{2}$, primers at $0.5 \mu \mathrm{M}$, and dNTPs at $200 \mu \mathrm{M}$ (final concentrations), with $0.75 \mathrm{U}$ of AmpliTaq Gold (Applied Biosystems) and $0.1 \mathrm{U}$ of Pwo DNA polymerase (Roche). PCR consisted of an initial incubation at $94^{\circ} \mathrm{C}$ for $10 \mathrm{~min}$, followed by 35 cycles of denaturation at $94^{\circ} \mathrm{C}$ for $30 \mathrm{~s}$, annealing for $30 \mathrm{~s}$, and extension at $72^{\circ} \mathrm{C}$ for $30 \mathrm{~s}$, with a final extension for $10 \mathrm{~min}$. The product was denatured at $95^{\circ} \mathrm{C}$ for $5 \mathrm{~min}$, followed by cooling at $1.5^{\circ} \mathrm{C} / \mathrm{min}$ to allow heteroduplex formation. Denaturing high performance liquid chromatography (DHPLC) analysis was carried out as described previously on a Wave 3500HT (Transgenomic). Fragments showing abnormal elution profiles were sequenced on the ABI PRISM 3100 DNA sequencer, employing Big Dye Terminator mix (Applied Biosystems). Sequence changes were confirmed by restriction digest.

In order to identify non-coding evolutionarily conserved regions, we first determined the orthologous regions in mouse, chicken and puffer fish (Takifugu) genomic sequences. These sequences were compared using VISTA (http://www.genome.lbl.gov/vista/index.shtml), and noncoding regions which showed conservation above $75 \%$ over a window of $100 \mathrm{bp}$ were selected. We compared selected regions with human genome-wide studies (Bertone et al. 2004) and sites of RNA polymerase II preinitiation complex (PIC) binding (Kim et al. 2005). By all these methods we identified a positive signal $61,100 \mathrm{bp} 5^{\prime}$ of the first known exon of GLI2. We designed oligonucleotide primers complementary to this sequence and sequence in exon 1, and used them for reverse transcriptase PCR with RNA isolated from fibroblasts from unaffected individuals. We found that one of these regions (primer pairs $5^{\prime}-\mathrm{CC}$ GCGCCAGCCAAGGTGGGATG-3' and 5'-AGCTACCG CTGCTGCTGCCGCTGCA-3' ${ }^{\prime}$ ) is spliced to the previously identified first exon of GLI2 suggesting that this locus represents a previously unrecognised exon of the human GLI2 transcript (Genbank Accession no. EF547128).

\section{Results}

The patient, a male currently 11 years old, was the only child born to healthy parents with no family history of limb malformations. The pregnancy was normal and he was born 
at 39 weeks of gestation. The limb abnormalities were apparent at birth and assessed in detail at the age of 6 years. The features were characteristic of SHFLD (see legend of Fig. 1). He had normal intelligence and was otherwise well, except that he developed a stricture of the urethral meatus, possibly attributable to balanitis xerotica obliterans that required a circumcision at the age of 2 years and was associated with secondary renal damage.

The G-banded karyotype revealed a de novo $\mathrm{t}(2 ; 18)(\mathrm{q} 14.2 ; \mathrm{p} 11.2)$ translocation in all cells analysed from a peripheral lymphocyte culture (Fig. 2a). Further characterisation of the translocation breakpoints was carried out by FISH. Four BAC clones from $2 \mathrm{q}$ and four from the $18 \mathrm{p}$ breakpoint region were selected from the human genome sequence (see section "Methods") and hybridised to metaphase chromosomes from the patient. Three of the $2 \mathrm{q}$ probes hybridised proximally to the $2 \mathrm{q}$ breakpoint on the $\operatorname{der}(2)$, while the remaining clone hybridised distally (data not shown), indicating that the breakpoint lay within a $1.5 \mathrm{Mb}$ interval. Four BACs located within this interval were selected for a secondary round of hybridisation, one of these (RP11-56H11) hybridised to the normal chromosome 2 and to both the $\operatorname{der}(2)$ and $\operatorname{der}(18)$ indicating that it spanned the breakpoint (Fig. 2b). These results confirmed that the $2 \mathrm{q}$ breakpoint lay within band q14.2. Of the chromosome 18 BACs, two hybridised proximally and two hybridised distally to the breakpoint; however, due to the success of the chromosome 2 hybridisation this was not further pursued. None of the clones hybridised with a reduced or absent signal on the derivative chromosomes, providing no evidence for a deletion associated with the translocation. This was further confirmed by array CGH of the patient's DNA and a reference
DNA to a high-density human $185 \mathrm{~K}$ genome-wide oligonucleotide array (average feature separation of $\sim 16 \mathrm{~kb}$ ). At this resolution, there were no consistent deviations from two genome copies identified in association with the translocation; in addition, no novel copy number variations were observed elsewhere in the genome (data not shown).

Further characterisation of the der(18) breakpoint by Southern analysis and subsequently by inverse PCR (see section "Methods") led to identification of the breakpoint at $120,960,699 \mathrm{bp}$ on chromosome 2 and at 10,368,893 bp on chromosome 18 (Fig. 3) (all nucleotide positions refer to the hg18 assembly). The breakpoint is very clean without the deletion of any nucleotides and the net addition of a single thymidine at one of the breakpoints (arbitrarily shown on the der(18) in Fig. 3b). Analysis of the normal sequences at the two breakpoints showed that both are unique, with no significant similarity except for a shared 3 bp TCA motif, indicating likely repair of the breaks by non-homologous end joining.

Neither breakpoint disrupts a known gene (Fig. 4). The $2 \mathrm{q} 14.2$ breakpoint lies between $I N H B B$ (135 kb away) and GLI2 (305 kb away) and within $172 \mathrm{~kb}$ of RALB, whilst the 18p11.22 breakpoint lies between APCDD1 (76 kb away) and VAPA (419 kb away). As outlined in the Introduction, we already favoured a more likely contribution of the 2q14.2 breakpoint to the SHFLD: the precise breakpoint mapping reinforced this for three reasons. First, several promising candidate genes lie near the $2 \mathrm{q} 14.2$ breakpoint. GLI2 is a mediator of Hedgehog signalling and Sonic hedgehog is a key regulator of normal limb patterning as Shh-null mice limb buds arrest in development soon after formation (Chiang et al. 1996). INHBB is a member of the
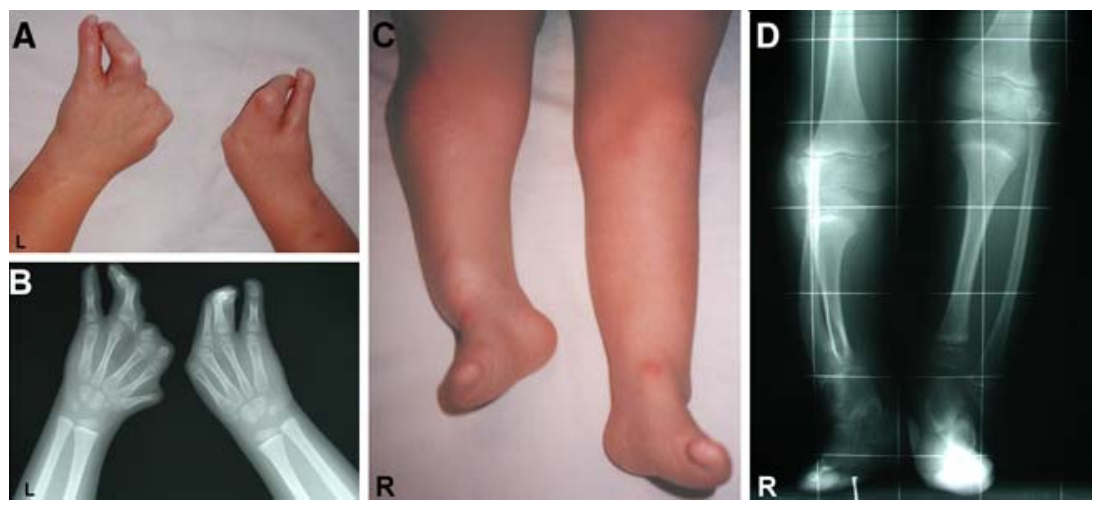

Fig. 1 Clinical phenotype and radiographic appearance of upper limbs (postoperative) (a, b) and lower limbs (preoperative) (c, d) of the translocation patient aged 6 years. a, b Appearance following bilateral release of syndactyly of the ulnar digits. Both hands exhibit oligodactyly, which is more severe on the radial side. On the right hand the first ray is entirely absent, all phalanges of the second digital ray are absent, the third ray has a single phalanx and the phalanges of the fourth and fifth rays are deformed. On the left side, the head of the first metacarpal is abnormal and the phalanges are absent. Rudimentary proximal pha- langes are present on the second and third digital rays and those of the fourth and fifth rays are deformed. The long bones of the forearm appear unaffected. c, $\mathbf{d}$ A single toe is present on each foot, there is also bilateral shortening of the tibiae, more marked on the right side, associated with a short, thick and bowed fibula leading to bowing of the leg and inversion of the foot. Additionally, bilateral patellar dislocation and distal flaring of the metaphases of the femur is present. $L$ left side; $R$ right side 
A

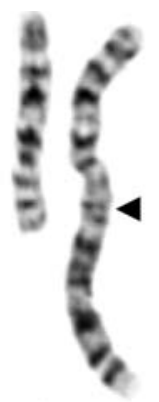

2

\section{B}

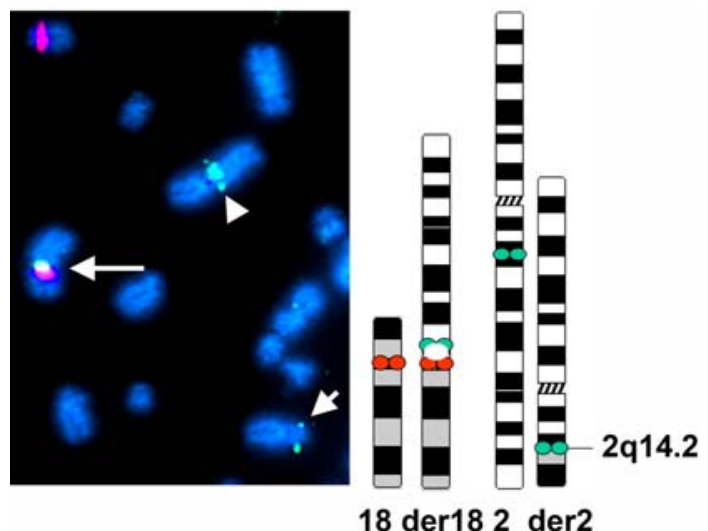

Fig. 2 a Partial G-banded karyotype of the patient showing a $\mathrm{t}(2 ; 18)(\mathrm{q} 14.2 ; \mathrm{p} 11.2)$ translocation. Arrowheads indicate the position of the breakpoints. b Fluorescence in situ hybridisation with bacterial artificial chromosomes $(B A C)$ clone RP11-56H11 from 2q14.2. The centromere of chromosome 18 is visualised as a red signal and the $\mathrm{BAC}$ as a green signal. Overlapping signal on der(18) appears white. Note hybridisation of the BAC to the normal chromosome 2 (arrowhead), der(2) (short arrow) and $\operatorname{der}(18)$ (long arrow). The BAC signal present both on $\operatorname{der}(2)$ and $\operatorname{der}(18)$ indicates that the clone spans the chromosome 2 translocation breakpoint

TGF- $\beta$ superfamily, a large group of extracellular proteins involved in many aspects of development; in the chick, $I n h b b$ is expressed during joint formation and ectopic application leads to abnormal chondrogenesis and joint fusion (Merino et al. 1999). RALB plays a role in mediating FGF signalling in Xenopus (Lebreton et al. 2003) and is expressed in the developing limb bud in mice (Zhao and Rivkees 2000). Second, the patient with a $(2 ; 4)(\mathrm{q} 14.2 ; \mathrm{q} 35)$ translocation described by Corona-Rivera et al. (2000) had holoprosencephaly as well as ectrodactyly; holoprosencephaly is known to result from heterozygous mutations of GLI2 (Roessler et al. 2003), suggesting that the 2q14.2 breakpoint in this patient was close to the GLI2 gene. Third, the homologous chromosomal bands in the mouse are 1E2 for the 2q14.2 breakpoint and bands 18E1 and 17E1 for the 18 p11.2 breakpoint. Interestingly, the spontaneous mouse mutant $D h$, for which the underlying mutation has not been identified, maps to band 1E2. Dh exhibits partial or complete loss of the tibia and preaxial digits, a pattern
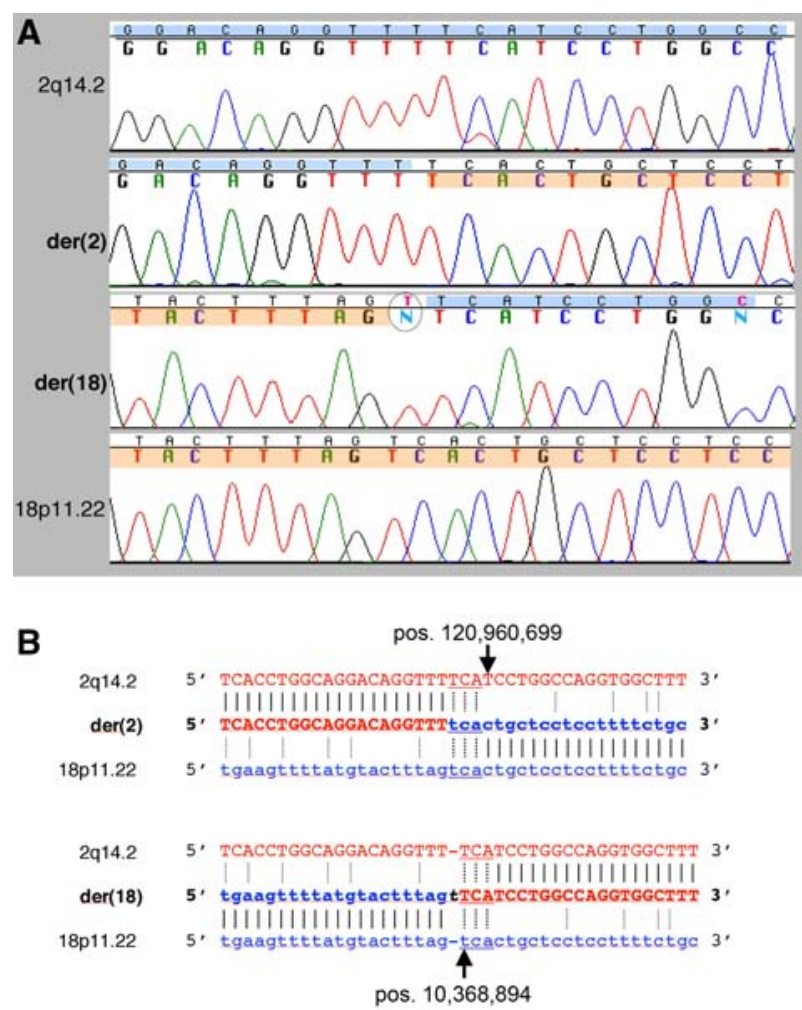

Fig. 3 DNA sequences from the two breakpoints of the $t(2 ; 18)$ (q14.2;p11.22). a Chromatograms showing breakpoint sequences compared with normal at the chromosome 2 and 18 breakpoints. b Alignment of the two normal and two breakpoint sequences. Red capital and blue lower case letters represent sequence from chromosomes 2 and 18, respectively; sequence on the derivative chromosomes is in bold. An extra thymidine residue (in black bold italic) is present at the breakpoint junction and the short homology between the chromosome 2 and 18 breakpoints at this junction is underlined

remarkably similar to the proband (Lettice et al. 1999). $D h$ was genetically indistinguishable from Gli2 in 561 meioses, although no Gli2 mutation was identified in $D h$ mice (Hughes et al. 1997).

To establish whether mutations in any of the genes in proximity to the chromosome 2 breakpoint (GLI2, INHBB, $R A L B$ or a fourth potential transcript, FLJ14816) underlie the ectrodactylous phenotype, we undertook mutation analysis by heteroduplex screening using Wave DHPLC in a panel of 44 SHFM, SHFLD and long bone deficiency patients (see section "Methods"). Analysis of GLI2 revealed three non-synonymous sequence changes in the patient panel. A $2159 \mathrm{G}>\mathrm{A}(\mathrm{R} 720 \mathrm{H})$ substitution was absent in 456 normal chromosomes but this could be discounted as a pathogenic mutation because the similarly affected mother did not show the change. A 4332_4333GC $>$ AT double nucleotide change encoding adjacent M1444I and L1445F substitutions in cis could also be discounted as pathogenic, both because it was inherited from the unaffected parent, and because the same double substitution 


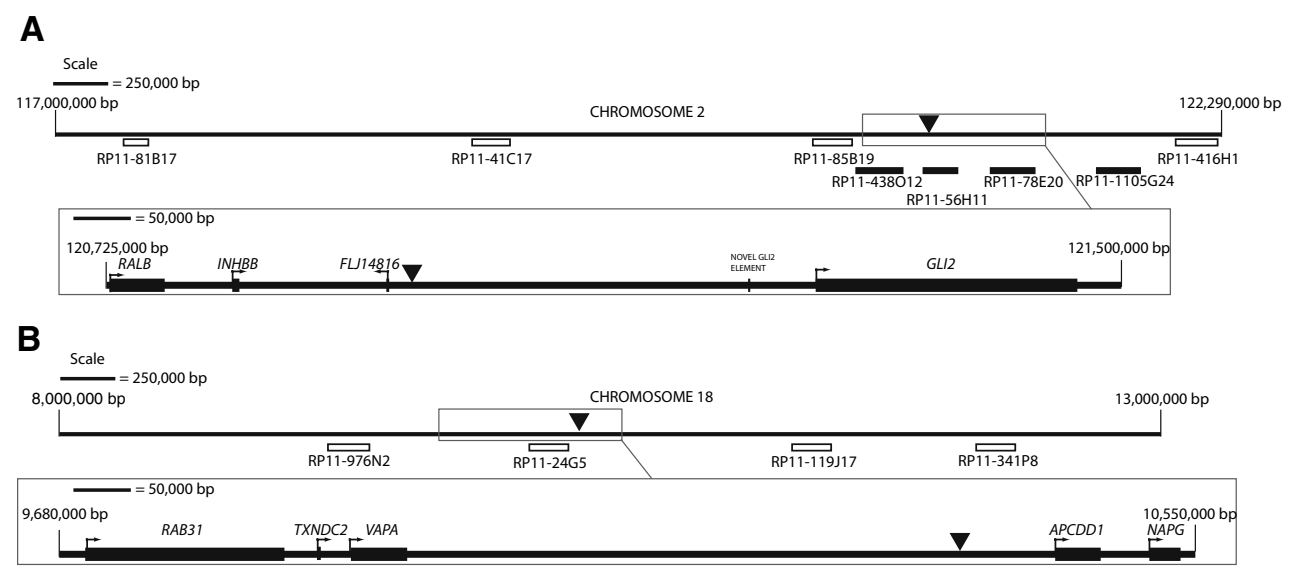

Fig. 4 Genomic maps of the regions around the $2 q$ breakpoint (a) and the $18 \mathrm{p}$ breakpoint (b). BACs used during the first (open rectangles) and second (filled rectangles) rounds of hybridisation are shown, the breakpoints are represented as arrowheads. The boxes enclose the re- gions shown at higher scale in the corresponding lower panels, including the genes surrounding the translocation breakpoints. The direction of transcription is represented by the arrows. The conserved region thought to represent the transcription start of GLI2 also shown (a) was identified in 2 out of 348 normal chromosomes. Finally, a 3349G $>\mathrm{T}$ (V1117L) substitution was absent in 566 normal chromosomes. The alteration was not seen in the unaffected mother and the unaffected father's DNA was unavailable for analysis, so we could not determine whether or not this change had arisen de novo. However, valine 1117 is not a highly conserved residue in the human GLI proteins nor in GLI2 proteins from other species. Analysis of $I N H B B$ and $R A L B$ did not reveal any coding changes in the patient cohort. Screening of a putative transcript (FLJ14816) located $\sim 20 \mathrm{~kb}$ from the breakpoint on chromosome 2 and predicted to comprise a single exon containing an open reading frame of 177 amino acids, was also negative.

To determine whether the translocation identified in this report affects the transcription levels of the four candidate genes in proximity to it, we screened these genes in the patient for informative polymorphisms. The proband was heterozygous for a novel T/C polymorphism in the $3^{\prime}$ untranslated region (UTR) of INHBB $(1956 \mathrm{~T}>\mathrm{C})$ (ss69374874) and for a known polymorphism (774T $>$ A) (rs1065518) in the 3' UTR of RALB. We amplified these transcripts from cDNA generated using RNA isolated from a fibroblast cell line derived from the patient. Amplification products were digested with a restriction endonuclease to discriminate between the alleles. Neither $R A L B$ nor $I N H B B$ showed a major difference in the expression levels of the normal and translocated alleles (data not shown). We could not amplify the predicted FLJ14816 transcript from cDNA derived from patient fibroblasts or an immortalised B-cell line, and neither the coding sequence nor the untranslated regions of GLI2 contained any informative polymorphisms in the proband.

\section{Discussion}

Although the limb represents a classical developmental system and the mechanisms of limb development have been described in considerable detail (Capdevila and Belmonte 2001; Tickle 2003), the pathogenesis of SHFLD/SHFM remains relatively poorly understood (Duijf et al. 2003). However, the primary defect appears to involve proximodistal outgrowth, whereas antero-posterior and dorso-ventral polarity is usually maintained. Proximo-distal outgrowth involves inductive interactions between the apical ectodermal ridge (AER) and the underlying progress zone (PZ). Failure to maintain the normal function of the AER has been described in some models of SHFM including the Dac mutant mouse, a model for SHFM3 (Crackower et al. 1998), and in Tp $73 l$ mutant mice (Yang et al. 1999; Mills et al. 1999). The induction of cell death in the AER by treatment with retinoic acid has also been shown to result in ectrodactyly (Sulik and Dehart 1988). Given that hemimelia may also accompany ectrodactyly, it is interesting that the posteriorly placed zone of polarising activity (ZPA) is also involved in AER maintenance. Clearly the identity of novel loci and/or mechanisms that disrupt limb development would be of considerable medical and biological importance. Some insight has been gained from the $D h$ mouse in which $F g f 4$ and $F g f 8$ are ectopically expressed in the anterior AER and this is associated with a reduction of the anterior mesenchyme (Lettice et al. 1999).

Here we have identified a novel chromosome translocation associated with SHFLD, and characterised its breakpoints in detail. Based on the previous description of a translocation with a 2q14.2 breakpoint associated with ectrodactyly, and the mapping of the $D h$ mouse mutant to a region of conserved synteny, we propose that $2 \mathrm{q} 14.2$ 
represents a novel locus for SHFLD. This SHFLD region contains several promising candidate genes, but despite screening these genes in a panel of patients we did not find any convincingly pathogenic mutations. There are several potential explanations for this. First, the clustering of $2 q 14.2$ breakpoints and SHFM could be a coincidence. We believe that this is unlikely given the additional evidence implicating this region in ectrodactyly (see sections "Introduction" and "Results"). Second, we may have missed rare pathogenic mutations because of patient selection or insensitivity of DHPLC screening. We cannot completely exclude this possibility, which can be investigated in future studies. Third, the limb malformation patients may have genomic rearrangements in this region of chromosome 2 . This possibility may be further investigated in the future. Fourth, the translocation may affect regulation of a nearby gene. We found no significant difference in the transcription levels of $I N H B B$ or RALB in patient fibroblasts; however, such an alteration may only be evident in temporal and/or tissue-specific developmental context. Additionally, we were unable to assay the affect of the translocation on GLI2 or FLJ14816. Therefore, misregulation of one or more candidate genes in this region during limb development remains a possible cause of malformations. The importance of regulatory mutations is well recognised (Kleinjan and van Heyningen 2005) and there are several examples of translocations that affect regulation of specific genes over distances of up to $1 \mathrm{Mb}$. For example, a translocation $1 \mathrm{Mb}$ away from $M A F$ is associated with cataract, ocular anterior segment dysgenesis and coloboma (Jamieson et al. 2002), microdeletions $900 \mathrm{~kb}$ proximal to POU3F4 cause X-linked deafness (de Kok et al. 1996), a deletion $1.2 \mathrm{Mb}$ away from $\mathrm{FOXCl}$ is associated with glaucoma/autosomal dominant iridogoniodysgenesis (Davies et al. 1999) and rearrangements up to $950 \mathrm{~kb}$ away from $\mathrm{SOX} 9$ are associated with campomelic dysplasia (Pfeifer et al. 1999). Identifying the regulatory sequences involved remains a major challenge, although there have been some notable successes, one being the ZPA regulatory sequence (ZRS) which lies $1 \mathrm{Mb}$ upstream of $S H H$ (Lettice et al. 2002). Interestingly, several other SHFM loci may also represent regulatory mutations: recent reviews of mapped SHFM patients suggest that $79 \%$ of SHFM1, 15-29\% of SHFM3 and $100 \%$ SHFM5 patients have chromosome abnormalities (Elliott and Evans 2006; Everman et al. 2006). Despite considerable efforts, the precise mechanisms of altered gene dosage remain to be defined at all these loci.

As discussed above, difficulties in associating cis regulatory elements with the genes they control often confounds efforts to implicate them in human disease. It seems likely that chromosomal breakage during evolution may be constrained by the requirement of cis regulatory elements to remain physically linked to the genes they act upon. A recent study has identified syntenic blocks on a wholegenome scale by generating multiple alignments of the human, mouse, chicken and frog genomes (Ahituv et al. 2005). Syntenic blocks are defined as chromosomal segments in which all sequences are in the same order and orientation in the species analysed, the minimum size of blocks included by Ahituv et al. (2005) was 200 kb. These evolutionarily conserved synteny blocks may delimit borders for distant cis regulatory regions and therefore conserved noncoding regions within them may harbour regulatory elements for the gene(s) in each block. A search of the synteny blocks reported by Ahituv et al. (2005) reveals that the 2 q14.2 translocation breakpoint reported here occurs at position $+1.04 \mathrm{Mb}$ in a block that extends over $1.77 \mathrm{Mb}$ in the human genome. This block is conserved in the mouse and chicken genomes, and is classified as within the largest $10 \%$ of such blocks, consistent with the presence of long-range cis regulatory elements. We conclude that any of the nine genes within this block (PTPN4, EPB41L5, FAM11B, $R A L B, I N H B B, F L J 14816, G L I 2, T F C P 2 L 1$, CLASP1), may be potentially affected by a regulatory mutation within the novel SHFLD region identified in this report.

Acknowledgments We are very grateful to the family for their help with this work. In addition the authors thank Han Brunnner, Hans van Bokhoven and Dominic Furniss for referring samples and for providing clinical information, Chris Ponting and Nicholas Dickens for assistance with bioinformatics, Samantha Knight and Regina Regan for performing CGH array analysis, Mary Elizabeth Laughridge for screening parental DNA samples and Bob Hill and Neil Dear for helpful discussions. This work was funded by a Wellcome Trust project grant (072761) and supported by Wellcome Trust programme grants to AOMW (004098 and 078666).

\section{References}

Ahituv N, Prabhakar S, Poulin F, Rubin EM, Couronne O (2005) Mapping $c i$-regulatory domains in the human genome using multispecies conservation of synteny. Hum Mol Genet 14:3057-3063

Bertone P, Stolc V, Royce TE, Rozowsky JS, Urban AE, Zhu X, Rinn JL, Tongprasit W, Samanta M, Weissman S, Gerstein M, Snyder M (2004) Global identification of human transcribed sequences with genome tiling arrays. Science 306:2242-2246

Capdevila J, Belmonte JCI (2001) Patterning mechanisms controlling vertebrate limb development. Annu Rev Cell Dev Biol 17:87-132

Celli J, Duijf P, Hamel BC, Bamshad M, Kramer B, Smits AP, Newbury-Ecob R, Hennekam RC, Van Buggenhout G, van Haeringen A, Woods CG, van Essen AJ, de Waal R, Vriend G, Haber DA, Yang A, McKeon F, Brunner HG, van Bokhoven H (1999) Heterozygous germline mutations in the p53 homolog p63 are the cause of EEC syndrome. Cell 99:143-153

Chiang C, Litingtung Y, Lee E, Young KE, Corden JL, Westphal H, Beachy PA (1996) Cyclopia and defective axial patterning in mice lacking Sonic hedgehog gene function. Nature 383:407-413

Corona-Rivera A, Corona-Rivera JR, Bobadilla-Morales L, GarciaCobian TA, Corona-Rivera E (2000) Holoprosencephaly, hypertelorism, and ectrodactyly in a boy with an apparently balanced de novo $\mathrm{t}(2 ; 4)$ (q14.2; 35 ). Am J Med Genet 90:423-426 
Crackower MA, Scherer SW, Rommens JM, Hui CC, Poorkaj P, Soder S, Cobben JM, Hudgins L, Evans JP, Tsui LC (1996) Characterization of the split hand/split foot malformation locus SHFM1 at 7q21.3-q22.1 and analysis of a candidate gene for its expression during limb development. Hum Mol Genet 5:571-579

Crackower MA, Motoyama J, Tsui LC (1998) Defect in the maintenance of the apical ectodermal ridge in the Dactylaplasia mouse. Dev Biol 201:78-89

Czeizel AE, Vitez M, Kodaj I, Lenz W (1993) An epidemiological study of isolated split hand/foot in Hungary, 1975-1984. J Med Genet 30:593-596

Davies AF, Mirza G, Flinter F, Ragoussis J (1999) An interstitial deletion of 6p24-p25 proximal to the FKHL7 locus and including AP2alpha that affects anterior eye chamber development. J Med Genet 36:708-710

de Kok YJ, Vossenaar ER, Cremers CW, Dahl N, Laporte J, Hu LJ, Lacombe D, Fischel-Ghodsian N, Friedman RA, Parnes LS, Thorpe P, Bitner-Glindzicz M, Pander HJ, Heilbronner H, Graveline J, den Dunnen JT, Brunner HG, Ropers HH, Cremers FP (1996) Identification of a hot spot for microdeletions in patients with $\mathrm{X}$ linked deafness type 3 (DFN3) $900 \mathrm{~kb}$ proximal to the DFN3 gene POU3F4. Hum Mol Genet 5:1229-1235

de Mollerat XJ, Everman DB, Morgan CT, Clarkson KB, Rogers RC, Colby RS, Aylsworth AS, Graham JM Jr, Stevenson RE, Schwartz CE (2003a) P63 mutations are not a major cause of non-syndromic split hand/foot malformation. J Med Genet 40:55-61

de Mollerat XJ, Gurrieri F, Morgan CT, Sangiorgi E, Everman DB, Gaspari P, Amiel J, Bamshad MJ, Lyle R, Blouin JL, Allanson JE, Le Marec B, Wilson M, Braverman NE, Radhakrishna U, Delozier-Blanchet C, Abbott A, Elghouzzi V, Antonarakis S, Stevenson RE, Munnich A, Neri G, Schwartz CE (2003b) A genomic rearrangement resulting in a tandem duplication is associated with split hand-split foot malformation 3 (SHFM3) at 10q24. Hum Mol Genet 12:1959-1971

Del Campo M, Jones MC, Veraksa AN, Curry CJ, Jones KL, Mascarello JT, Ali-Kahn-Catts Z, Drumheller T, McGinnis W (1999) Monodactylous limbs and abnormal genitalia are associated with hemizygosity for the human 2q31 region that includes the HOXD cluster. Am J Hum Genet 65:104-110

Duijf PH, van Bokhoven H, Brunner HG (2003) Pathogenesis of splithand/split-foot malformation. Hum Mol Genet 12(Spec No 1):R51-R60

Elliott AM, Evans JA (2006) Genotype-phenotype correlations in mapped split hand foot malformation (SHFM) patients. Am J Med Genet A 140:1419-1427

Emami-Ahari Z, Mahloudji M (1974) Bilateral absence of the tibias in three sibs. Birth Defects Orig Artic Ser 10:197-200

Everman DB, Morgan CT, Lyle R, Laughridge ME, Bamshad MJ, Clarkson KB, Colby R, Gurrieri F, Innes AM, Roberson J, Schrander-Stumpel C, van Bokhoven H, Antonarakis SE, Schwartz CE (2006) Frequency of genomic rearrangements involving the SHFM3 locus at chromosome 10q24 in syndromic and non-syndromic split-hand/foot malformation. Am J Med Genet A 140:1375-1383

Goodman FR, Majewski F, Collins AL, Scambler PJ (2002) A 117-kb microdeletion removing HOXD9-HOXD13 and EVX2 causes synpolydactyly. Am J Hum Genet 70:547-555

Hughes DC, Allen J, Morley G, Sutherland K, Ahmed W, Prosser J, Lettice L, Allan G, Mattei MG, Farrall M, Hill RE (1997) Cloning and sequencing of the mouse Gli2 gene: localization to the Dominant hemimelia critical region. Genomics 39:205-215

Ianakiev P, Kilpatrick MW, Dealy C, Kosher R, Korenberg JR, Chen XN, Tsipouras P (1999) A novel human gene encoding an F-box/ WD40 containing protein maps in the SHFM3 critical region on 10q24. Biochem Biophys Res Commun 261:64-70
Ianakiev P, Kilpatrick MW, Toudjarska I, Basel D, Beighton P, Tsipouras $\mathrm{P}$ (2000) Split-hand/split-foot malformation is caused by mutations in the p63 gene on 3q27. Am J Hum Genet 67:59-66

Jamieson RV, Perveen R, Kerr B, Carette M, Yardley J, Heon E, Wirth MG, van Heyningen V, Donnai D, Munier F, Black GC (2002) Domain disruption and mutation of the bZIP transcription factor, $\mathrm{MAF}$, associated with cataract, ocular anterior segment dysgenesis and coloboma. Hum Mol Genet 11:33-42

Kapur S, Remes GM, Waterman DF, Toriello HV, Higgins JV (1982) Two families with tibial aplasia and ectrodactyly. Am J Hum Genet 34:96A

Kim TH, Barrera LO, Zheng M, Qu C, Singer MA, Richmond TA, Wu Y, Green RD, Ren B (2005) A high-resolution map of active promoters in the human genome. Nature 436:876-880

Kleinjan DA, van Heyningen V (2005) Long-range control of gene expression: emerging mechanisms and disruption in disease. Am J Hum Genet 76:8-32

Lebreton S, Boissel L, Moreau J (2003) Control of embryonic Xenopus morphogenesis by a Ral-GDS/Xral branch of the Ras signalling pathway. J Cell Sci 116:4651-4662

Lettice L, Hecksher-Sorensen J, Hill RE (1999) The dominant hemimelia mutation uncouples epithelial-mesenchymal interactions and disrupts anterior mesenchyme formation in mouse hindlimbs. Development 126:4729-4736

Lettice LA, Horikoshi T, Heaney SJ, van Baren MJ, van der Linde HC, Breedveld GJ, Joosse M, Akarsu N, Oostra BA, Endo N, Shibata M, Suzuki M, Takahashi E, Shinka T, Nakahori Y, Ayusawa D, Nakabayashi K, Scherer SW, Heutink P, Hill RE, Noji S (2002) Disruption of a long-range cis-acting regulator for Shh causes preaxial polydactyly. Proc Natl Acad Sci USA 99:7548-7553

Majewski F, Kuster W, ter Haar B, Goecke T (1985) Aplasia of tibia with split-hand/split-foot deformity. Report of six families with 35 cases and considerations about variability and penetrance. Hum Genet 70:136-147

Merino R, Macias D, Ganan Y, Rodriguez-Leon J, Economides AN, Rodriguez-Esteban C, Izpisua-Belmonte JC, Hurle JM (1999) Control of digit formation by activin signalling. Development 126:2161-2170

Mills AA, Zheng B, Wang XJ, Vogel H, Roop DR, Bradley A (1999) p63 is a p53 homologue required for limb and epidermal morphogenesis. Nature 398:708-713

Naveed M, Nath SK, Gaines M, Ali-Ali MT, Al-Khaja N, Hutchings D, Golla J, Deutsch S, Bottani A, Antonarakis SE, Ratnamala U, Radhakrishna U (2007) Genomewide linkage scan for split-hand/ foot malformation with long-bone deficiency in a large Arab family identifies two novel susceptibility loci on chromosome 1q42.2-q43 and 6q14.1. Am J Hum Genet 80:105-111

Pfeifer D, Kist R, Dewar K, Devon K, Lander ES, Birren B, Korniszewski L, Back E, Scherer G (1999) Campomelic dysplasia translocation breakpoints are scattered over $1 \mathrm{Mb}$ proximal to SOX9: evidence for an extended control region. Am J Hum Genet 65:111-124

Roessler E, Du YZ, Mullor JL, Casas E, Allen WP, Gillessen-Kaesbach G, Roeder ER, Ming JE, Ruiz i Altaba A, Muenke M (2003) Loss-of-function mutations in the human GLI2 gene are associated with pituitary anomalies and holoprosencephaly-like features. Proc Natl Acad Sci USA 100:13424-13429

Scherer SW, Poorkaj P, Massa H, Soder S, Allen T, Nunes M, Geshuri D, Wong E, Belloni E, Little S, Zhou L, Becker D, Kere J, Ignatius J, Nllkawa N, Fukushlma Y, Hasegawa T, Weissenbach J, Boncinelli E, Trask B, Lap-Chee Tsui LC, Evans JP (1994) Physical mapping of the split hand/split foot locus on chromosome 7 and implication in syndromic ectrodactyly. Hum Mol Genet $3: 1345-1354$

Schinzel A (2001) Catalogue of unbalanced chromosome aberrations in man, 2nd edn. Walter de Gruyter, Berlin 
Sidow A, Bulotsky MS, Kerrebrock AW, Birren BW, Altshuler D, Jaenisch R, Johnson KR, Lander ES (1999) A novel member of the F-box/WD40 gene family, encoding dactylin, is disrupted in the mouse dactylaplasia mutant. Nat Genet 23:104-107

Sulik KK, Dehart DB (1988) Retinoic-acid-induced limb malformations resulting from apical ectodermal ridge cell death. Teratology $37: 527-537$

Tickle C (2003) Patterning systems-from one end of the limb to the other. Dev Cell 4(4):449-458

van Bokhoven H, Hamel BC, Bamshad M, Sangiorgi E, Gurrieri F, Duijf PH, Vanmolkot KR, van Beusekom E, van Beersum SE, Celli J, Merkx GF, Tenconi R, Fryns JP, Verloes A, NewburyEcob RA, Raas-Rotschild A, Majewski F, Beemer FA, Janecke A, Chitayat D, Crisponi G, Kayserili H, Yates JR, Neri G, Brunner
HG (2001) p63 Gene mutations in EEC syndrome, limb-mammary syndrome, and isolated split hand-split foot malformation suggest a genotype-phenotype correlation. Am J Hum Genet 69:481-492

Yang A, Schweitzer R, Sun D, Kaghad M, Walker N, Bronson RT, Tabin C, Sharpe A, Caput D, Crum C, McKeon F (1999) p63 is essential for regenerative proliferation in limb, craniofacial and epithelial development. Nature 398:714-718

Zhao Z, Rivkees SA (2000) Tissue-specific expression of GTPas RalA and RalB during embryogenesis and regulation by epithelialmesenchymal interaction. Mech Dev 97:201-204

Zlotogora J (1994) On the inheritance of the split hand/split foot malformation. Am J Med Genet 53:29-32 